\title{
Validity and Reliability Study of Patient Satisfaction Level- Questionnaire (UNS-BsQ8) for Breast Fibroadenoma Patient after Surgery
}

\author{
Muhammad David Perdana Putra ${ }^{1}$, Kristanto Yuli Yarsa ${ }^{2}$, Mudib ${ }^{1}$, Zhafirah Ramadhanty ${ }^{3}$ \\ ${ }^{1}$ Department of Surgery, Faculty of Medicine, Sebelas Maret University, Surakarta, Indonesia \\ 2 Department of Surgery, Oncology Division, Sebelas Maret University, Surakarta, Indonesia \\ ${ }^{3}$ Medical of Faculty, Sebelas Maret University, Surakarta, Indonesia
}

\section{ARTICLE INFO}

Received : 31 January 2020

Reviewed : 02 March 2020

Accepted : 28 May 2020

Keywords:

breast fibroadenoma, questionnaire, reliability, validity

${ }^{*}$ Corresponding author:

Muhammad David Perdana Putra

Department of Surgery, Faculty of

Medicine, Sebelas Maret University,

Surakarta

guitarist.bvb@gmail.com

\author{
A BSTRACT
}

\begin{abstract}
Background: This study aimed to develop a patient satisfaction level-questionnaire (UNS-BsQ8) for breast fibroadenoma patients in Indonesia and to test the reliability and validity of the questionnaire by using established models.
\end{abstract}

\begin{abstract}
Methods: A total of 83 women with breast fibroadenoma completed the questionnaire after their surgeries. We assessed the construct validity and the reliability by referring to its Pearson's $r$ table value and Cronbach's $\alpha$ coefficient.
\end{abstract}

Results: It showed that 83 subjects completed all 8 questions of the instrument between January 1-31, 2020; the Pearson's value of all items of the UNS-BsQ8 with the lowest value of $r=.398$ in question number 8 , followed by $r=.411$ in question number 7 with an $r$-value of $r>.1584$ indicated that these questions were valid. Cronbach's $\alpha$ coefficient of $>.60$ indicated questionable internal consistency.

Conclusions: The result of this study proves that UNS-BsQ8 is accepted, valid, and reliable. It can be used for future investigation, especially in patients undergoing minimally invasive surgery.

\section{INTRODUCTION}

Fibroadenoma is one of the most common benign tumors of the breast in the adolescent. They count for $69 \%$ of all breast masses and $44-94 \%$ of all biopsied breast lesions. Endoscopic surgery, vacuum-assisted percutaneous biopsy, and ablative procedure are the procedure options to remove fibroadenoma up to 3 $\mathrm{cm}$ in size [1]. However, there are still only a few researches regarding their quality of life in Indonesia $[2,3]$. Patient satisfaction level measurement should reflect the domains including the physical, social, and cultural environments, and it is common but also able to show the uniqueness of all subjects [4]. The questionnaire is a generic measure that could be applied in any adult population. A Standard questionnaire is ideally valid and reliable, which means that the measurement reflects the concept and is consistent [5].

In Indonesia, the validity and reliability of the instrument are still on development, especially in breast fibroadenoma patients. A few validity and reliability tests of the Indonesian version of the Patient Satisfaction Survey have been done in a few groups of benign patients in Germany [6]. However, differences between Germany and Indonesian languages, traditions, and cultures require the development of a needs assessment questionnaire specifically for Indonesian patients [7].

This study aimed to develop a patient satisfaction level-questionnaire (UNS-BsQ8) for breast fibroadenoma patients in Indonesia and to test the reliability and validity of the questionnaire by using established models.

\section{METHODS}

\begin{abstract}
Patients
Each subject either filed an online form or underwent an interview by phone, chat, or face to face interview in the Oncology Outpatient Clinic of Kasih Ibu Hospital. A total of 91 community members were contacted between January 1-31, 2020, and selected by the following inclusion criteria: (1) at least 30 years of age, (2) able to complete the questionnaire by themselves, and (3) having no communication problem. The exclusion criteria are having a degenerative disease and/or mental
\end{abstract}


illness and malignant disease. Among 91 community members, only 83 agreed to participate in this study and completed the questionnaire.

This cross-sectional study was ethically approved by The Health Research Ethics Committee of Faculty of Medicine of Sebelas Maret University on June 27, 2018, no. 118/UN27/6/KEPK/2018.

\section{Instrument}

The UNS-BsQ8 was a designed combination of ordinal scaled (grading scale from 1 - poor to 5 - excellent). To measure patient satisfaction, we administered the patient satisfaction questionnaire.

Table 1. UNS-BsQ8 design

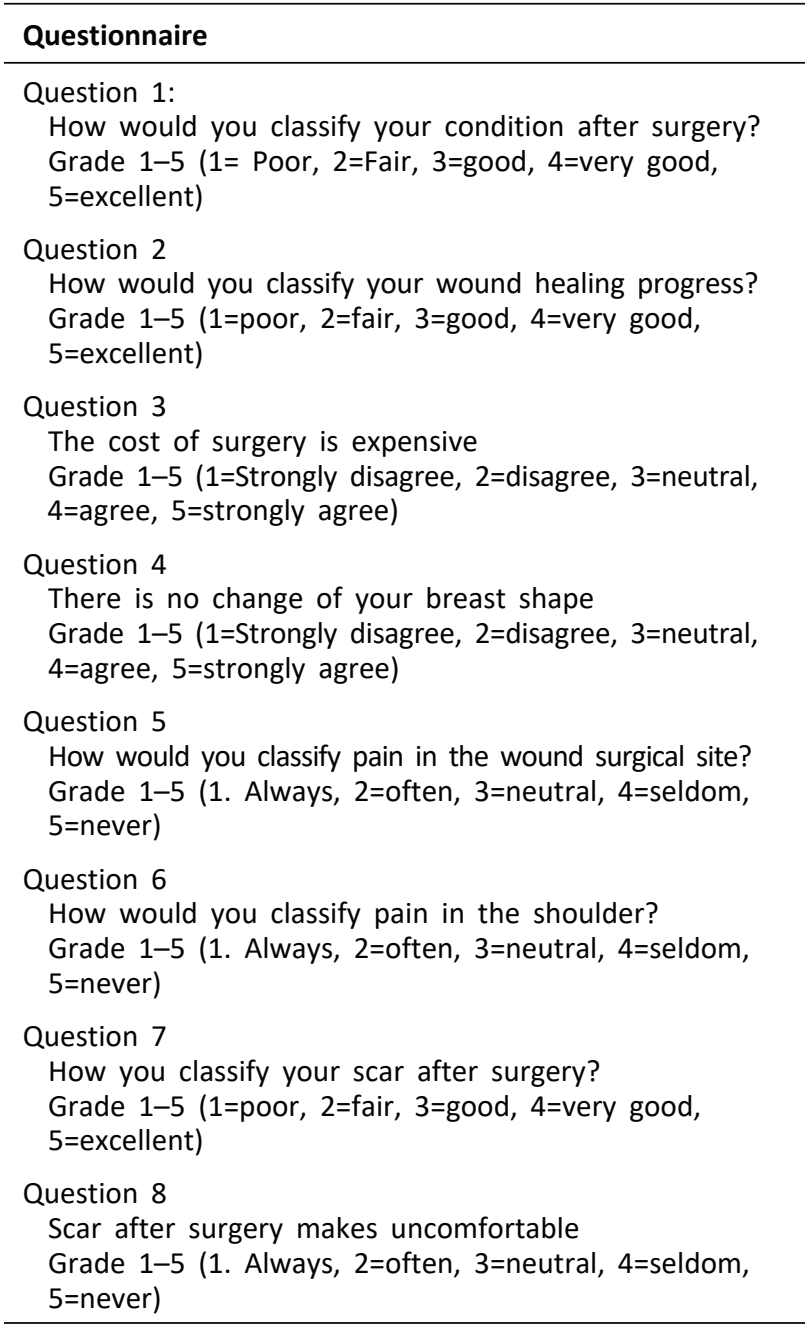

From Table 1, questions number 1 and 2 described the wound healing satisfaction level, questions 3 describe cost satisfaction monitor, questions 4 about breast shape after surgery, questions 5 and 6 the pain after surgery level, and questions 7 and 8 scar formation after surgery satisfaction level.

The UNS-BsQ8 is easily scored and consists of 8 items designed to measure patient satisfaction with services. Each item shows a score of 1 to 5 . Final scoring is conducted by summing up the individual item scores to produce a range of 8 to 40 with higher scores representing greater satisfaction (satisfied $=40-30$, neutral $=29-20$, unsatisfied $=19-8)$. Validity and Reliability tests were also conducted, and, in conclusion, the questionnaire can be used in patients with breast fibroadenoma after surgery.

\section{Statistical Analysis}

In this cross-sectional study, a minimum of 40 samples is needed to conduct validity and reliability tests. Construct validity was measured by referring to Pearson's $r$ table correlation value, and the reliability was measured by referring to Cronbach's alpha coefficient. The value of Pearson's $r$ table must be $>.1548$ and Cronbach's alpha coefficient $>.60$.

The demographical questions were also asked the respondents including age and educational level. The data were collected and then analyzed by using IBM SPSS Statistics 25 for Windows.

\section{RESULTS}

A total of 40 patients underwent surgery between 2017-2019 came to Oncology Outpatient Clinic from January 1-31, 2020.

Table 2. Subject characteristics

\begin{tabular}{lcc}
\hline Characteristic & Frequency (n = 40) & (\%) \\
\hline Age & 28 & \\
$<30$ & 12 & 70 \\
$>31$ & & 30 \\
Education Level & 6 & \\
Senior High School & 10 & 15 \\
Diploma & 18 & 25 \\
Bachelor Degree & 6 & 45 \\
Master Degree & 15 \\
\hline
\end{tabular}

Table 2 shows the characteristics of the respondents in this study. All the respondents were female with a mean age of 24 years old. Based on the educational status, most of the respondents graduated from bachelor's degree (45\%) and diploma (35.96\%).

Table 3 shows Pearson's $r$ value and Cronbach's alpha coefficient for all 8 questions. The Pearson's value of all items of the UNS-BsQ8 shows $r>.1548$ with the lowest value of $r=.398$ in item number 8 , followed by $r=.411$ in item number 7 . Other than that, all items show the value of $r>.300$. The Cronbach's alpha coefficient in all items shows a value of $>.600$, between the range of 0.606 and 0.609 , supporting the internal consistency of the item. 
Table 3. Pearson's $r$ value and Cronbach's alpha coefficient

\begin{tabular}{lcc}
\hline Scale & Pearson's $r$ value & Cronbach's Alpha \\
\hline Question 1 & 0.624 & 0.606 \\
Question 2 & 0.593 & 0.614 \\
Question 3 & 0.524 & 0.664 \\
Question 4 & 0.412 & 0.683 \\
Question 5 & 0.536 & 0.658 \\
Question 6 & 0.455 & 0.645 \\
Question 7 & 0.411 & 0.662 \\
Question 8 & 0.358 & 0.669 \\
\hline
\end{tabular}

The mean age of participants was 24 years with 19 years old as the youngest and 38 years old as the oldest participant. In a large population of another study in 265,402 women, the mean age of development of breast fibroadenoma included the young age $(<30$ years old) [1].

The validation of UNS-BsQ8 was performed by evaluating the construct validity and reliability, referring to Pearson's $r>.1548$ and Cronbach's alpha > .60. All items showed $r>.1548$ so that all items of UNS-BsQ8 were valid and reflected the concepts. The Cronbach's alpha showed a value of $>.60$ ranging around 0.606-0.669. It means that all items had questionable internal consistency.

Despite all questions that showed the value of $r>.1548$, some questions had a lower value than the others. Question number 8 (Scar after surgery) had the lowest value, showing $r=.398$. From the previous study, patients might experience postoperative cosmetic deformity or secondary asymmetry, especially after the removal of fibroadenoma [8]. Another question that had a quite low value than the others $(r=.411)$ was question number 7 (classify their scar). All those questions were associated with scar formation questionnaires, and this low value might be caused by the respondents having a hard time translating what they exactly felt and the lack of information for reconstructive surgery and at subsequent follow-up appointments [9]. Overall, based on Pearson's $r$ and Cronbach's alpha values, all questions were valid and reliable and could be used as an instrument to assess the patient satisfaction level especially in patients with benign breast tumors.

This study also had some limitations. Validity and reliability tests for other quality of life instruments analyzed the validity by referring to not only its Pearson's $r$-value but also other validity tests such as convergent and discriminant validity, known-group validity, and factor analysis [6]. Further validity and reliability tests in other groups are also needed.

\section{CONCLUSIONS}

The result of this study proves that UNS-BsQ8 is an accepted, valid, and reliable tool for the patient with breast fibroadenoma after surgery. The usage of UNS-BsQ8 in recent years has already delivered meaningful data that may serve as a benchmark for future investigation especially patients undergoing minimally invasive surgery.

\section{DECLARATIONS}

\section{Competing of Interest}

The authors declare no competing interest in this study.

\section{Acknowledgment}

We thank all community members, Dr. dr. Kristanto Yuli Yarsa, Sp.B(K)Onk., and friends, and all people that helped to participate in this study.

\section{REFERENCES}

1. Soltanian $H$, Lee $M$. Breast fibroadenomas in adolescents: current perspectives. Adolesc Health Med Ther. 2015;6:159-69.

2. Ferlay J, Soerjomataram I, Dikshit R, et al. Cancer incidence and mortality worldwide: Sources, methods and major patterns in GLOBOCAN 2012. Int J Cancer. 2015;136(5):E359-E386.

3. Kementrian Kesehatan Republik Indonesia. Pedoman Nasional Pelayanan Kedokteran Kanker Payudara. Komite Penanggulangan Kanker Nasional; 2017.

4. Verdugo MA, Schalock RL, Keith KD, Stancliffe RJ. Quality of life and its measurement: Important principles and guidelines. J Intellect Disabil Res. 2005;49(10):707-17.

5. Feeny DH, Eckstrom E, Whitlock EP, Perdue LA. A primer for systematic reviewers on the measurement of functional status and health-related quality of life in older adults. Rockville (MD): Agency for Healthcare Research and Quality (US); 2013.

6. Eller A, Janka $R$, Lux $M$, et al. Stereotactic vacuumassisted breast biopsy (VABB) - A patients' survey. Anticancer Res. 2014;34(7):3831-37.

7. Li L, Xue J, Li ZZ, Chen LZ. Development and testing of a reliable and valid patient-needs questionnaire for breast cancer inpatients in China. J Clin Diagnostic Res. 2016;10(10):XC06-XC10.

8. Cerrato F, Labow Bl. Diagnosis and management of fibroadenomas in the adolescent breast. Semin Plast Surg. 2013;27(1):23-5.

9. Gobbi D, Dall'Igna P, Alaggio R, Nitti D, Cecchetto G. Giant fibroadenoma of the breast in adolescents: Report of 2 cases. J Pediatr Surg. 2009;44(2):e39-e41. 